\title{
Comparison of the Pattern of Metastatic Spread of Squamous Cell Cancer and Adenocarcinoma of the Uterine Cervix
}

\author{
Charles W. Drescher, M.D., Michael P. Hopkins, M.D., and James A. Roberts, M.D. ${ }^{1}$ \\ Department of Obstetrics and Gynecology, University of Michigan Hospitals, 1500 East Medical Center Drive, D2246 Medical Professional \\ Building, Ann Arbor, Michigan 48109-0718 \\ Received November 9, 1987
}

\begin{abstract}
Retrospective review of medical records and autopsy findings in patients dying of squamous cell cancer or adenocarcinoma of the uterine cervix was undertaken to evaluate for possible differences in biologic behavior between these tumor types. Twenty-one patients with each tumor type were evaluated. Patients with adenocarcinoma were found to have a higher incidence of tumor involvement of the paraaortic lymph nodes $(13 / 21$ vs $6 / 20, P<0.05)$, uterine corpus $(17 / 17$ vs $12 / 20, P<0.05)$, and adrenal gland $(7 / 21$ vs $0 / 21, P<0.005)$. Presence of ascites $(9 / 21$ vs $2 / 21, P<0.05)$ and hydrothorax $(9 / 21$ vs $3 / 21, P<0.05)$ was also significantly more frequent in patients with adenocarcinoma. These findings suggest that this tumor may behave differently in regard to pattern of metastatic spread or response to therapy. The therapeutic implications of these findings deserve further study. क) 1989 Academic Press, Inc.
\end{abstract}

The reported incidence of adenocarcinoma of the uterine cervix varies from 4 to $20 \%$ of all cervical malignancies, with most studies suggesting the true incidence to be near 5\% [1-3]. Many studies suggest that the prognosis for patients with adenocarcinoma may be worse than that for patients with squamous cell cancer [4,5]. Proposed explanations have included differences in their patterns of growth and metastasis as well as sensitivity to radiotherapy. Although autopsy studies have helped to delineate the patterns of tumor spread and recurrence for squamous cell cancer [6] comparatively little autopsy information has been reported for cervical adenocarcinoma. To more completely evaluate for differences in tumor behavior we reviewed the medical records and autopsy reports of patients dying from adenocarcinoma of the uterine cervix and compared them with information obtained from patients dying of cervical squamous cell cancer.

${ }^{1}$ To whom requests for reprints should be addressed.

\section{MATERIALS AND METHODS}

Study patients were identified by review of the University of Michigan Medical Center (UMMC) tumor registry from 1936 through 1985 for patients dying of cervical squamous cell or adenocarcinoma and who had autopsy performed at UMMC. A total of 165 patients were identified, including 26 patients with adenocarcinoma and 139 patients with squamous cell cancer. Medical records and autopsy reports were reviewed for information on tumor stage, type of therapy received, immediate cause of death, and anatomic location of tumor at autopsy. Histologic slides of tumors were reviewed and classified according to previously reported criteria [7]. Briefly, histologic features considered consistent with invasive adenocarcinoma included complex endocervical glandular architecture with areas of epithelial atypia and evidence of invasion through cervical stroma. Associated areas of in situ carcinoma were considered conclusive evidence of origin in endocervical glands or surface epithelium. Any case in which uncertainty existed as to the site of origin was excluded from analysis. Squamous cell cancer was diagnosed using standard criteria. Only patients with pure squamous cell cancer or adenocarcinoma were included in this report.

All autopsies were performed at the University of Michigan Medical Center by resident pathologists under the supervision of staff physicians. A consistent autopsy protocol was utilized throughout the study period and the necropsy data were recorded on a standardized form. Areas examined for tumor were divided into four groups: lymph nodes, pelvis, abdomen, and miscellaneous. Nodal tissue examined included paraaortic, paratracheal, scalene, inguinal, axillary and retroperitoneal pelvic lymph nodes (RPLN). The RPLN group comprises nodal tissue identified within the retroperitoneal tissue of the true pelvis and includes the external iliac, internal iliac, obturator, 
and paracervical nodal tissue. The autopsy protocol at the University of Michigan does not provide for individual dissection of each of these nodal tissues. Pelvic sites included vulva, vagina, uterus, ovary, bladder, rectum, and ureter. The abdominal group included intraabdominal organs (adrenal glands, colon, gallbladder, kidneys, liver, omentum, pancreas, stomach, spleen, small bowel) as well as ascites and peritoneal studding. Ascites was defined as the presence of free intraperitoneal fluid of any amount. Peritoneal studding was considered present only when diffuse involvement of the abdominal and pelvic peritoneum was noted. The miscellaneous group includes intrathoracic sites such as lung and pleura and hydrothorax (defined as free intrapleural fluid of any amount), as well as bone, skin, and central nervous system tissues.

Comparisons between groups were made using a maximum likelihood $\chi^{2}$ test $[8]$.

\section{RESULTS}

Twenty-six patients with cervical adenocarcinoma were identified. Five patients were excluded, leaving 21 patients who ultimately composed this group. Three excluded patients were diagnosed and treated for cervical adenocarcinoma but subsequently died of a second primary carcinoma and had no evidence of residual cervical cancer at autopsy. Second primary tumors in these patients included squamous cell carcinomas of the jaw, lung, and vagina. An additional patient was diagnosed with synchronous primary adenocarcinomas of the cervix and colon, and although metastatic lesions of both primaries were identified at autopsy they could not be clearly separated histologically in all circumstances. The final patient excluded had previously undergone a supracervical hysterectomy for endometrial adenocarcinoma. She subsequently developed adenocarcinoma of the cervical stump, which appeared histologically to be of endocervical origin; however, the possibility of recurrent endometrial carcinoma could not be excluded with certainty.

A comparison group of 21 patients with squamous cell cancer was selected from the initial 139 by attempting to match as closely as possible tumor stage and date of diagnosis with patients in the adenocarcinoma group. The average year of diagnosis (mean \pm SD) was 1959 \pm 12 and $1966 \pm 12$ for patients with adenocarcinoma and squamous cell cancer, respectively. The incidence of nulliparity and average age at diagnosis were $20 \%$ $(3 / 15)$ and 49 years for patients with adenocarcinoma and $22 \%(4 / 18)$ and 50 years for patients with squamous cell disease.

Stage of disease and subsequent therapy received are indicated in Tables 1 and 2. Two-thirds of patients in each group had either stage III or stage IV disease. Four patients (two from each group) received no therapy. Rea-
TABLE 1

FIGO Stage of Cervical Cancer

\begin{tabular}{lcc}
\hline \multicolumn{2}{c}{ Histologic type } \\
\cline { 2 - 3 } Stage & Squamous cell cancer & Adenocarcinoma \\
\hline I & 2 & 3 \\
II & 5 & 4 \\
III & 9 & 9 \\
IV & 5 & 5 \\
Total & 21 & 21 \\
\hline
\end{tabular}

sons for failure to treat included patient desire, underlying medical illness, or advanced disease. Radiation therapy was the most commonly used form of treatment and was utilized in 37 of 38 treated patients, including 14 patients with adenocarcinoma and 17 patients with squamous cell cancer who received radiation as the sole treatment modality. External beam and brachytherapy were utilized in all but four patients who received external beam treatment alone. Combination radiation and surgical therapy was utilized in four patients with adenocarcinoma and two patients with squamous cell cancer. Chemotherapy was utilized in six patients after other treatment options had failed. This included two patients with squamous cell carcinoma and four patients with adenocarcinoma.

Data regarding immediate cause of death are shown in Table 3. Obstructive renal disease and sepsis were the most frequent events resulting in death in both groups and accounted for $66 \%$ of deaths of known causes.

Location of residual disease at the time of autopsy is shown in Table 4. RPLN involvement was noted in $61.2 \%$ of patients with squamous cell carcinoma and in $75 \%$ of patients with adenocarcinoma. Paraaortic and paratracheal nodal involvement was more frequently noted in patients with adenocarcinoma; however, this was statistically significant only at the paraaortic site $(13 / 21$ vs $6 / 20, P<$ $0.05)$. Scalene nodes were involved in one patient in each group while inguinal or axillary nodal involvement was not noted in any patient.

TABLE 2

Therapy Received

\begin{tabular}{lccccccccc}
\hline & \multicolumn{3}{c}{ Squamous cell cancer } & & \multicolumn{4}{c}{ Adenocarcinoma } \\
Therapy & I $^{a}$ & II & III & IV & & I & II & III & IV \\
\hline RTX & 1 & 4 & 7 & 5 & & 4 & 6 & 4 \\
SURG & & & & & 1 & & & \\
R\&S & 1 & & 1 & & 2 & & 2 & \\
None & & 1 & 1 & & & & 1 & 1 \\
Total & 2 & 5 & 9 & 5 & 3 & 4 & 9 & 5 \\
\hline
\end{tabular}

a Stage.

${ }^{b}$ RTX, radiation; SURG, surgery; R\&S, radiation and surgery. 
TABLE 3

Causes of Death

\begin{tabular}{lcc}
\hline Cause of death & Squamous cell cancer & Adenocarcinoma \\
\hline Obstructive renal failure & 6 & 7 \\
Sepsis & 5 & 6 \\
Pneumonia & 2 & 1 \\
Cachexia & 1 & 2 \\
Hemorrhage & 1 & \\
DIC & 1 & 2 \\
Pulmonary embolism & 1 & 2 \\
Diabetes mellitus & 4 & 1 \\
Other & 21 & 21 \\
Unknown & 21 & \\
Total &
\end{tabular}

TABLE 4

Location of Tumor at Autopsy

Squamous

Group Site $\quad \begin{aligned} & \text { Squamous } \\ & \text { cell cancer }\end{aligned}$ Adenocarcinoma $P$

Lymph node

Axillary
Inguinal
Paraaortic
Paratracheal
RPLN
Scalene

0
0
$6^{a}$
4
13
1

0

Lymp node

Pelvis

Bladder
Ovaries
Rectum
Ureter
Uterus
Vagina
Vulva

Abdomen

Adrenal

Ascites

Colon

Gallbladder

Kidney

Liver

Pancreas

Peritoneum

Omentum

Small Bowel

Spleen

Miscellaneous

Stomach

Bone

CNS

Hydrothorax

Lung

Pleura

Skin

\footnotetext{
${ }^{a} N=20$

${ }^{\circ} N=17$.
}

Residual disease in the pelvis was a common finding in patients with both squamous cell cancer and adenocarcinoma. Involvement of the uterine corpus was noted in all patients with adenocarcinoma who did not have their uterus removed as part of therapy, whereas disease in the uterus was noted in 12 of 20 patients with squamous cell carcinoma. This difference was statistically significant $(17 / 17$ vs $12 / 20, P<0.05)$. Vaginal, ovarian, bladder, rectal, and ureteral involvement was found with approximately equal frequency in each group. Vulvar involvement was not noted in either group.

Liver was the most frequently involved intraabdominal organ and was noted to contain tumor in approximately one-third of all patients. Involvement of pancreas, spleen, stomach, gallbladder, and kidney was noted only rarely. There was no difference in frequency of involvement of these organs between groups. Adrenal gland involvement was noted in 7 of 21 patients with adenocarcinoma and in none of 21 patients with squamous cell $(P<0.005)$. Ascites was found more frequently in patients with adenocarcinoma $(9 / 21$ vs $2 / 21, P<0.05)$. Clinically significant ascites (i.e., $>500 \mathrm{ml}$ ) was noted in $45 \%$ of those patients with ascites, including four patients with adenocarcinoma and one patient with squamous cell cancer. Disseminated peritoncal involvement was noted twice as frequently in patients with adenocarcinoma $(6 / 21$ vs $3 / 21)$ but this difference was not statistically significant. Omental and small and large bowel involvement was noted in approximately one-fifth of patients and there was no difference in involvement of these organs between groups.

Tumor involvement of lung parenchyma was noted in 7 of 21 patients with squamous cell cancer and 9 of 21 patients with adenocarcinoma $(P=$ n.s. $)$. Involvement of the pleural cavity was noted less frequently in both groups, being found in 3 of 21 squamous cell carcinoma patients and 5 of 21 patients with adenocarcinoma $(P=$ n.s.). Hydrothorax was noted significantly more frequently in patients with adenocarcinoma $(9 / 21$ vs $3 / 21, P<0.05)$. The presence of hydrothorax was strongly associated with the simultaneous presence of ascites. All nine patients with hydrothorax in the adenocarcinoma group also had ascites, whereas ascites was found in only one of three patients with hydrothorax in the squamous cell cancer group. The volume of the hydrothorax exceeded $300 \mathrm{ml}$ in three patients with adenocarcinoma and one patient with squamous cell cancer.

\section{DISCUSSION}

Retrospective study of necropsy data obtained in a consistent fashion offers a reliable method for determining the extent of malignant disease. Although the influence of confounding variables, such as differences in treatment modalities or host response, cannot be completely ex- 
cluded, the information obtained in this study suggests that important differences in biologic behavior exist between squamous cell cancer and adenocarcinoma of the uterine cervix.

Previous authors have suggested that cervical adenocarcinoma may be relatively resistant to radiotherapy, and improved survival with combination radiation and surgical therapy over radiation therapy alone has been demonstrated in some reports. Berek et al. [4] found residual tumor in $21 \%$ of surgical specimens from patients with stage I and II disease previously treated with radiation, while Prempree et al. [9] noted a greater tendency toward local and regional recurrences in patients treated with radiation therapy alone. In the present study residual disease was found in the uterine corpus in all patients with adenocarcinoma who did not undergo hysterectomy as part of therapy. Although four patients with residual tumor in the uterus had stage II disease, the majority had more advanced stage with large tumors, and poor control with radiation in these patients could be anticipated based on the large initial tumor volume. At present the role of adjunctive surgical therapy is unclear, particularly in patients with advanced disease. One cannot conclude from the data presented in this study that the addition of hysterectomy will improve survival. Residual disease following radiation therapy within the uterine corpus occurred significantly less frequently in patients with squamous cell cancer.

It has been suggested that adenocarcinoma has a propensity for endophytic growth into the cervical stroma [10], which could allow early access to lymphatic channels and result in disseminated nodal metastasis. Although such a pattern of growth has been suggested for patients with squamous cell cancer with barrel-shaped cervical lesions, the higher incidence of paraaortic nodal metastasis noted in patients with adenocarcinoma suggests that adenocarcinoma may have a greater tendency for this type of spread.

Of particular interest was the high frequency $(7 / 21)$ of adrenal gland involvement in the patients with adenocarcinoma compared to patients with squamous cell cancer $(0 / 21)$. Henriksen [6], in an extensive autopsy study of 356 patients with cervical carcinoma, noted a $1.6 \%$ incidence of adrenal gland involvement. However, this included 14 patients with adenocarcinoma who were not separated from patients with squamous cell cancer. The tendency for adenocarcinoma to metastasize to the adrenal gland with moderate frequency may indicate a propensity toward hematogenous spread, possibly along the ovarian vessels. Additionally, the adrenal gland may provide a local hormonal environment that is favorable for the growth of metastatic implants of adenocarcinoma. Metastases to the adrenal gland never appeared to be clinically significant.

Ascites and hydrothorax were more common among patients with adenocarcinoma and they usually occurred simultaneously. The malignant characteristic of this fluid in the present study cannot be assured as cytologic studies were not performed; however, contamination with malignant cells seems highly likely as many of these patients had gross tumor within the abdominal or pleural cavities. Of additional interest was the higher frequency of disseminated peritoneal involvement noted in patients with adenocarcinoma, although this did not reach statistical significance. The tendency toward peritoneal spread and the formation of ascitic fluid suggests that intraabdominal spread may be an important route of metastasis for cervical adenocarcinoma.

This report summarizes autopsy findings in patients dying of adenocarcinoma of the uterine cervix and compares them to data obtained from patients with squamous cell cancer. Several differences were noted that suggest these tumors behave differently in regard to patterns of spread and/or response to treatment. The therapeutic implications of these findings are unclear at present; however, they may be more clearly defined with future study.

\section{REFERENCES}

1. Gusberg, S. B., and Corscaden, J. A. The pathology and treatment of adenocarcinoma of the cervix, Cancer 4, 1066-1072 (1951).

2. Baker, H. W., Brach, C. B., and Dickson, R. J. Adenocarcinoma of the cervix uteri, Obstet. Gynecol. 4, 664-669 (1954).

3. Rutledge, F. N., Galakatos, A. E., Wharton, J. T., and Smith, J. P. Adenocarcinoma of the uterine cervix, Amer. J. Obstet. Gynecol. 122, 236-245 (1975).

4. Berek, J. S., Castaldo, T. W., Hacker, N. F., Petrilli, E. S., Lagasse, L. D., and Moore, J. G. Adenocarcinoma of the uterine cervix, Cancer 48, 2734-2741 (1981).

5. Moberg, P. J., Einhorn, N., Silfversward, C., and Soderberg, G. Adenocarcinoma of the uterine cervix, Cancer 57, 407-410 (1986).

6. Henriksen, E. The lymphatic spread of carcinoma of the cervix and the body of the uterus, Amer. J. Obstet. Gynecol. 58, 924942 (1949).

7. Abell, M. R., and Gosling, J. R. Gland cell carcinoma of the uterine cervix, Amer. J. Obstet. Gynecol. 83, 729-755 (1962).

8. Sokal, R. R., and Rohlf, F. J. Biometry. Freeman (1981).

9. Prempree, T., Amornmarn, R., and Wizenberg, M. J. A therapeutic approach to primary adenocarcinoma of the cervix, Cancer 56, 1264-1268 (1985).

10. Knapp, R. C., and Berkowitz, R. S. Gynecologic oncology, Macmillan, New York (1986). 